\title{
BMJ Open Protocol for a randomised feasibility study of Point-Of-care HIV viral load testing to Enhance Re-suppression in South Africa: the POwER study
}

Jienchi Dorward (D) ,1,2 Yukteshwar Sookrajh, ${ }^{3}$ Hope Ngobese, ${ }^{3}$ Richard Lessells, ${ }^{2,4}$ Fathima Sayed, ${ }^{2}$ Elliot Bulo, ${ }^{3}$ P Moodley, ${ }^{5,6}$ Natasha Samsunder, ${ }^{2}$ Lara Lewis, ${ }^{2}$ Sarah Tonkin-Crine (1) , ${ }^{1,7}$ Paul K Drain,,${ }^{8,9,10}$ Gail Hayward (1) , ${ }^{1}$ Christopher C Butler (D) , ${ }^{1}$ Nigel Garrett (D) ${ }^{2,11}$

To cite: Dorward J, Sookrajh Y, Ngobese $\mathrm{H}$, et al. Protocol for a randomised feasibility study of Point-Of-care HIV viral load testing to Enhance Re-suppression in South Africa: the POwER study. BMJ Open 2021;11:e045373. doi:10.1136/ bmjopen-2020-045373

- Prepublication history and additional material is published online only. To view please visit the journal online (http://dx.doi. org/10.1136/bmjopen-2020045373).

Received 29 September 2020 Revised 21 January 2021 Accepted 02 February 2021
Check for updates

(C) Author(s) (or their employer(s)) 2021. Re-use permitted under CC BY. Published by BMJ.

For numbered affiliations see end of article.

Correspondence to Dr Jienchi Dorward; jienchi.dorward@phc.ox.ac.uk

\section{ABSTRACT}

Introduction Access to HIV viral load testing remains difficult for many people on antiretroviral therapy (ART) in low-income and middle-income countries. Weak laboratory and clinic systems often delay the detection and management of viraemia, which can lead to morbidity, drug resistance and HIV transmission. Point-of-care testing could overcome these challenges. We aim to assess whether it is feasible to conduct a randomised trial of point-of-care viral load testing to manage viraemia. Methods and analysis We will conduct an openlabel, single-site, individually randomised, feasibility study of Point-Of-care HIV viral load testing to Enhance Re-suppression, in Durban, South Africa. We will enrol approximately 100 people living with HIV who are aged $\geq 18$ years, receiving first-line ART but with recent viraemia $\geq 1000$ copies $/ \mathrm{mL}$, and randomise them $1: 1$ to receive point-of-care viral load or standard laboratory viral load monitoring, after 12 weeks. All participants will continue to receive care from public sector healthcare workers following South African HIV management guidelines. Participants with persistent viraemia $\geq 1000$ copies $/ \mathrm{mL}$ will be considered for switching to second-line ART. We will compare the proportion in each study arm who achieve the primary outcome of viral suppression $<50$ copies $/ \mathrm{mL}$ at 24 weeks after enrolment. Additional outcomes include proportions retained in the study, proportions with HIV drug resistance, time to viral load results and time to switching to second-line ART. We will assess implementation of point-of-care viral load testing using process evaluation data, and through interviews and focus groups with healthcare workers.

Ethics and dissemination University of Oxford Tropical Research Ethics Committee and the Biomedical Research Ethics Committee of the University of KwaZulu-Natal have approved the study. We will present results to stakeholders, and through conferences and open-access, peer-reviewed journals.

Trial registration number PACTR202001785886049.

\section{INTRODUCTION}

Initiating antiretroviral therapy (ART) to achieve viral load suppression among all

\section{Strengths and limitations of this study}

- The Point-Of-care HIV viral load testing to Enhance Re-suppression (POwER) study will provide new evidence to guide the development of interventions using point-of-care HIV viral load testing to improve the management of viraemia in low-income and middle-income countries.

- The study will be conducted in a public health facility and will inform the utility and ease of implementation of point-of-care viral load testing in a routine clinical setting.

- The study is limited by a moderate sample size and limited power to detect an effect of point-of-care viral load testing on viral resuppression.

people with HIV is crucial to achieve the Joint United Nations Programme on HIV/AIDS target of ending AIDS by 2030. However, poor adherence to ART and/or HIV drug resistance can lead to HIV viraemia, with associated increases in morbidity, mortality, onward HIV transmission and the development and spread of HIV drug resistance. ${ }^{1}$ Viral load testing can identify viraemia and guide adherence counselling, and/or switching to second-line ART regimens if HIV drug resistance is suspected. However, in low-income and middle-income countries (LMICs), the majority of ART is provided in primary care, where there is limited laboratory viral load capacity, weak clinic systems to act on viral load results and a paucity of evidence-based interventions to improve adherence. ${ }^{1-5}$ Poor adherence can be due to complex social and psychological issues (eg, gender-based violence, alcohol use, migrant labour and long distances to clinics) ${ }^{6}$ Therefore, multiple visits for blood draws and result review confers an additional burden for these 
vulnerable populations. Laboratory viral load results are often not reviewed until the next routine clinic visit after several months, by which time adherence problems may have worsened. ${ }^{1-4}$

Point-of-care viral load testing could allow same-day adherence counselling and switching to second-line ART, and more efficient, person-centred care by reducing the burden of clinic visits for review of blood results; thereby leading to faster viral load resuppression. WHO has approved the Xpert HIV-1 VL (Cepheid, Sunnyvale, USA) ${ }^{7}$ and the m-PIMA HIV-1/2 VL (Abbott, Chicago, USA) ${ }^{8}$ as accurate, quantitative point-of-care HIV viral load assays for use in LMICs. However, these assays have not yet been rolled out for ART monitoring in LMICs, due to concerns around implementation, and with limited randomised trial evidence to support clinical effectiveness. To date there is only one, single-site, randomised trial that has evaluated the clinical effectiveness of these assays. This was the (Simplifying HIV Treatment and Monitoring) STREAM point-of-care viral load trial, conducted by our team, in which 390 adults stable on ART for 6 months were randomised to receive either usual care with laboratory viral load testing, or care by an enrolled nurse and point-of-care viral load testing. ${ }^{9}$ After 12 months, people in the intervention arm were more likely to have been referred into a community ART delivery programme, and had $14 \%$ higher retention in care and viral suppression. ${ }^{10}$ However, the combined intervention was provided by research staff, meaning we do not know if point-of-care viral load assays can be implemented effectively in public sector clinics in LMICs. ${ }^{11}$ Furthermore, STREAM only enrolled clinically stable patients ( $5 \%$ had viraemia at enrolment), and so there is inadequate data on the effect of point-of-care viral load testing among people with viraemia who are a vulnerable, priority population.

\section{OBJECTIVES}

The overall aim of this feasibility study ${ }^{12}$ is to provide exploratory estimates to guide the development of a future larger trial of point-of-care testing to manage HIV viraemia. Our specific objectives are to:

1. Broadly estimate the effect size of point-of-care viral load testing compared with standard laboratory viral load testing on viral resuppression $<50$ copies/mL after 24 weeks.

2. Determine the feasibility of recruiting, randomising and following up patients in a randomised trial of point-of-care viral load testing to manage HIV viraemia.

3. Assess the perceptions of staff and the practical changes in clinic systems that are required to implement point-of-care viral load testing for a subset of patients in a primary care clinic in South Africa.

\section{METHODS AND ANALYSIS}

\section{Trial design}

This will be a an open-label, single-site, individually randomised, feasibility study of point-of-care viral load testing for the management of HIV viraemia (figure 1). We aim to enrol approximately 100 adults receiving first-line ART and who have viraemia, and randomise them in a 1:1 ratio to receive point of care versus standard laboratory-based viral load testing. We will assess viral suppression after 24 weeks in each arm, and estimate recruitment, fidelity of the intervention and retention in care. We will also assess the implementation of point-of-care viral load testing using process evaluation data, in-depth interviews and focus group discussions.

\section{Study setting}

The study will take place at the Prince Cyril Zulu Clinic; a large public sector clinic run by the eThekwini Municipality Health Unit in central Durban, South Africa. The clinic manages approximately 11000 people receiving ART and follows South African National Department of Health guidelines. ${ }^{13}$ Since December 2019, South Africa has started replacing current non-nucleoside reverse transcriptase inhibitors (NNRTIs), with the new drug dolutegravir for first-line ART. ${ }^{1415}$ Dolutegravir is an integrase strand transfer inhibitor that, in high-income settings, has been found to have better tolerability, efficacy and durability than regimens based on NNRTIs such as efavirenz. ${ }^{1416}$ Current first-line ART regimens at Prince Cyril Zulu Clinic are therefore tenofovir disoproxil fumarate, emtricitabine and efavirenz, or tenofovir disoproxil fumarate, lamivudine and dolutegravir. ${ }^{13}{ }^{14}$ Viral load

Non-pregnant, HIV-positive adults aged $\geq 18$

years, receiving first-line ART, with the latest viral load in the past 6 weeks $\geq 1000$ copies/ml

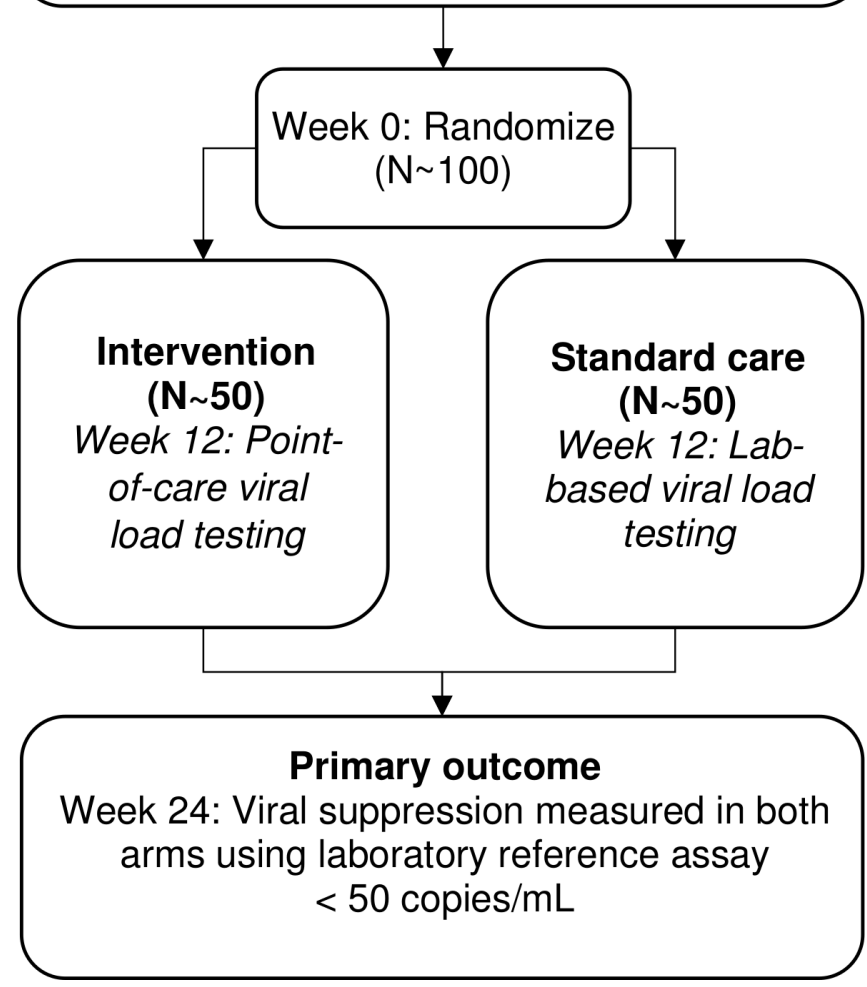

Figure 1 Consort diagram of the POwER study. ART, antiretroviral therapy. 
testing is performed at six and twelve months after ART initiation and annually thereafter. Similar to the WHO guidelines, patients with viraemia are given enhanced adherence counselling, with a repeat viral load within 3 months. If viraemia persists on NNRTI-based regimens, then HIV drug resistance is more likely, and so a switch to second-line ART should be considered. ${ }^{13}{ }^{17}$ For dolutegravir-based regimens, HIV drug resistance is less likely, and so a switch to second-line ART is only recommended if there are signs of clinical or immunological failure, or after 2 years of persistent viraemia. ${ }^{13}$

\section{Eligibility criteria}

Eligible patients will be HIV-positive adults aged $\geq 18$ years, receiving first-line NNRTI-based or dolutegravirbased ART, with the latest viral load in the past 6 weeks $\geq 1000$ copies $/ \mathrm{mL}$, and with no previous enhanced adherence counselling for this episode of viraemia. Pregnant women will not be eligible as they are routinely referred out of the Prince Cyril Zulu Clinic for antenatal care.

\section{Study procedures \\ Recruitment}

Potential participants will be identified by clinic staff using laboratory reports, electronic clinic information systems and clinic notes.

\section{Informed consent, screening and enrolment}

A research assistant or nurse will describe the study to potential participants, address any questions and seek written informed consent for participation. A research nurse will then assess eligibility using the patient's demographics, and a brief medical history and examination, including pregnancy testing for all women.

\section{Randomisation and blinding}

A statistician will generate the allocation sequence using random numbers generated in SAS V.9.4 (SAS Institute). We will stratify randomisation by first-line ART regimen at enrolment (NNRTI-based or dolutegravir based). The allocation sequence will contain variable block sizes, with participants randomised in a 1:1 ratio to the intervention or standard of care arm. All study staff apart from the statistician and data manager will be blinded to the allocation sequence. The allocation sequence will be programmed into the REDCap V.8.7.1 (Vanderbilt University, Nashville, USA $)^{18}$ enrolment electronic case report form (eCRF). At enrolment, the research assistant or research nurse will complete the enrolment eCRF, which will automatically assign the intervention allocation. As this is an open label study, study staff, clinic staff and participants will not be blinded to intervention allocation.

\section{Baseline assessments}

At enrolment, a nurse will administer a baseline sociodemographic questionnaire, and take a clinical history and examination, including an ART history. Throughout the study, South African Department of Health guidelines for managing viraemia will be used. ${ }^{13}$ This includes enhanced adherence counselling, which will be performed by clinic staff with participants in both arms using the preenrolment viral load result. Techniques used during the counselling include ART education, evaluation of social support, mental health screening and the use of treatment supporters where appropriate. ${ }^{19} \mathrm{~A}$ nurse will also draw blood for routine tests for investigation of viraemia, which currently include CD4 count testing, hepatitis B surface antigen, creatinine and haemoglobin (table 1$){ }^{13}$

\section{Follow-Up}

Participants in both arms will have routine clinic visits scheduled at the healthcare worker and participant's discretion, which is typically every 28 days to coincide with ART collection. At these visits, clinic staff will provide ongoing enhanced adherence guidelines, clinical support and ART when necessary. South African guidelines recommend a repeat viral load 3 months after the first high result $\geq 1000$ copies $/ \mathrm{mL}$. As this study is taking place within a routine care setting, we anticipate that not all participants will attend within the 3-month visit window. In these cases, we will allow repeat viral load to be done at another time, at the attending clinician's discretion.

\section{Viral load testing interventions}

For participants in the point-of-care arm, the repeat viral load test will be performed by a nurse, phlebotomist or laboratory technician in the clinic, using a WHO approved, fully automated point-of-care viral load assay (such as the Xpert HIV-1 VL). Assay run times are $\leq 90 \mathrm{~min}$. Clinic staff will be encouraged to provide results in the same clinic visit to inform ongoing management. If the participant cannot wait, or results are not available in the same visit, they will be available at the next clinic visit scheduled at staff and participant's discretion.

In the standard of care arm, samples for viral load testing will be transported on the same day to the National Health Laboratory Service (NHLS) for testing using their routine laboratory assays (eg, Alinity m HIV-1 assay (Abbott, Chicago, USA)). Results are normally available after 7 days and will be provided to the participant at the next clinic visit, arranged at the participants and healthcare worker's discretion (typically after 7-28 days for results, depending on the participant's availability, ART supply and clinic schedules).

\section{Management of viral load results at routine follow-up clinic visits}

Viral load results will be managed by clinic staff in accordance with South African guidelines, which include guidance for management of both efavirenz and dolutegravir-based regimens, and do not advise routine HIV drug resistance testing. ${ }^{20}$ Participants with a viral load $\geq 1000$ copies $/ \mathrm{mL}$ may meet criteria for viral failure (two consecutive viral loads $\geq 1000$ copies $/ \mathrm{mL}$ over 3 months apart) and will be considered for a switch to second-line ART. Switching to second-line ART and the choice of regimen will be at the clinician's discretion and will depend on the participant's first-line ART regimen, previous ART history, participant's preferences, perceived 
Table 1 Schedule of evaluation in the POwER study

\begin{tabular}{|c|c|c|c|c|c|c|c|}
\hline \multirow[b]{2}{*}{ Weeks in study } & \multirow{2}{*}{$\begin{array}{l}\text { Enrol } \\
0\end{array}$} & \multicolumn{5}{|c|}{ Routine clinic visit follow-up* } & \multirow{2}{*}{$\begin{array}{l}\text { Study exit } \\
24 \dagger\end{array}$} \\
\hline & & 4 & 8 & 12 & 16 & 20 & \\
\hline Informed consent & $\mathrm{x}$ & & & & & & \\
\hline Locator information & $\mathrm{x}$ & & & & & & \\
\hline Demographics & $x$ & & & & & & \\
\hline Medical history & $\mathrm{x}$ & & & & & & \\
\hline Vital signs & $x$ & $\mathrm{x}$ & $\mathrm{x}$ & $\mathrm{x}$ & $\mathrm{x}$ & $\mathrm{x}$ & $\mathrm{x}$ \\
\hline Physical examination & $\mathrm{x}$ & & & & & & \\
\hline Urine pregnancy test & $\mathrm{x}$ & & & & & & \\
\hline Eligibility screen & $\mathrm{x}$ & & & & & & \\
\hline Enhanced adherence counselling & $x$ & $\mathrm{x}$ & $\mathrm{x}$ & $\mathrm{x}$ & $X \ddagger$ & $X \ddagger$ & $X \ddagger$ \\
\hline Randomisation & $\mathrm{x}$ & & & & & & \\
\hline Routine Department of Health testing§ & $x$ & & & & & & \\
\hline POC viral load (intervention arm) & & & & $\mathrm{x} \boldsymbol{1}$ & & & \\
\hline Laboratory viral load (standard of care arm) & & & & Xף & & & \\
\hline Reference viral load outcome measure & & & & & & & $\mathrm{x}$ \\
\hline Evaluation of new POC assays ${ }^{* *}$ & $x$ & & & $\mathrm{x}$ & & & $\mathrm{x}$ \\
\hline Stored blood and HIV drug resistance testing†† & $\mathrm{x}$ & & & $\mathrm{X}$ & & & $\mathrm{X}$ \\
\hline Stored urine for ART drug level testing & $\mathrm{x}$ & & & & & & \\
\hline
\end{tabular}

*Scheduled at participants and healthcare worker's discretion but typically every 28 days.

†Study exit visit window 22-30 weeks.

fOngoing enhanced adherence counselling for participants with viral load $\geq 50$ copies $/ \mathrm{mL}$.

$\S C D 4$ count testing, hepatitis $B$ surface antigen, creatinine and haemoglobin.

ПRepeat viral load testing recommended at 12 weeks but may be done any time during follow-up at the attending clinician's discretion.

${ }^{* *}$ Maximum of $8 \mathrm{~mL}$ venous blood and/or a finger-prick capillary blood sample.

††Retrospective drug resistance testing for viraemic participants at enrolment, follow-up or study exit (10 mL EDTA).

ART, antiretroviral therapy; POC, point-of-care; POwER, Point-Of-care HIV viral load testing to Enhance Re-suppression.

adherence and current guidelines. ${ }^{20}$ Participants with a second viral load $<1000$ copies/mL will remain on firstline ART. In accordance with guidelines for the transition to dolutegravir, those receiving an NNRTI may have this switched to a dolutegravir-based first-line regimen. ${ }^{13}$

Data capture during routine clinic visits

Research staff will monitor participants' clinical records to capture visit dates, whether enhanced adherence counselling was conducted by a counsellor, nurse or doctor, medication prescriptions including ART and laboratory results.

\section{Study exit visit}

The study exit visit will occur at 24 weeks (visit window 22-30 weeks), after which participants will continue receiving standard care at the Prince Cyril Zulu Clinic. At 24 weeks, any participant who has not attended the study exit visit will be called by the research team and asked to attend, irrespective of whether they have a routine clinic visit scheduled in the exit visit window.

\section{Participant reimbursement}

We will reimburse study participants ZAR150 (approximately £8.00) after the enrolment visit and ZAR100
(£5.50) after the 24-week visit, to cover any additional time spent at the clinic and any inconvenience due to study participation. ${ }^{21}$

\section{Extended follow-up of routine data}

For participants who provide additional consent, research staff may access routine medical records for up to 5 years after the study exit visit, in order to determine longer term retention in care, ART adherence and viral load results. For participants who are lost to follow-up, and who provided consent at enrolment, the participants mortality status may be checked on the South African National Population Register.

\section{Additional laboratory specimens}

Participants who provide additional consent will also have $10 \mathrm{~mL}$ of venous blood taken for storage and retrospective ART drug level and HIV drug resistance testing. Plasma will be stored at enrolment, during follow-up alongside the repeat viral load test, and at study exit. In addition, at enrolment, $5 \mathrm{~mL}$ of urine will be taken for storage and ART drug-level testing. We may use study samples to validate new point-of-care viral load, HIV drug resistance or 
Table 2 Study objectives and outcomes in the power study

\section{Objectives}

\section{Primary objective}

To estimate the effect of implementing point-ofcare viral load testing versus standard laboratory viral load testing on the proportion of HIV positive participants with viraemia who subsequently achieve viral resuppression

\section{Secondary objectives}

To assess whether it is feasible to perform a randomised implementation trial of point-of-care viral load testing to manage viraemia in a routine South African clinic, by determining:

\section{(A) What proportion of people with HIV viraemia is feasible to enrol?}

(B) What proportion of those enrolled is feasible to follow-up?

(C) What proportion of point-of-care viral load results are received and acted on in the same day?

\section{Outcome measures}

The proportion of participants in each arm with viral load $<50$ copies/mL measured on laboratory reference assay
Time point(s) of evaluation of this outcome measure (if applicable)

24 weeks after enrolment

\section{Tertiary Objectives}

To estimate the effect of point-of-care viral load testing versus standard laboratory viral load testing on:

\section{(A) Time to detection of viral failure (consecutive viral loads $\geq 1000$ copies $/ \mathrm{mL}$ ) in each arm \\ (B) Time to switch to second-line ART in each arm \\ (C) Appropriate switching to dolutegravir in each arm}

\section{(D) HIV drug resistance in each arm}

\section{Qualitative study objectives:}

What changes in clinic systems are required and what are the views and experiences of staff in implementing point-of-care viral load testing to manage viraemia?
(A) Proportion of patients with viraemia at the study clinic who are successfully enrolled in the study

(B) Proportion of enrolled participants who attend the study exit visit overall and in each arm

(C1) The proportion of point-of-care viral load tests that are communicated to participants on the same day and (C2) The proportion of point-of-care viral load tests $\geq 1000$ copies $/ \mathrm{mL}$ that result in same day enhanced adherence counselling and (C3) The proportion of point-of-care viral load tests $\geq 1000$ copies $/ \mathrm{mL}$ that result in same day switch to second-line ART
(A) During study enrolment period

(B) 24 weeks after enrolment

(C) 12 weeks after enrolment when point-of-care testing is performed

(A) Days from enrolment to availability of viral (A) By 24 weeks after enrolment load result $\geq 1000$ copies $/ \mathrm{mL}$

(B) Days from enrolment to appropriate switching to second-line ART, among participants with viral failure

(B2) By 24 weeks after enrolment

(C) Time to appropriate switching to

(C3) By 24 weeks after enrolment dolutegravir in each arm

$\begin{array}{ll}\text { (D) The proportion of participants in each } & \text { (D4) } 24 \text { weeks after enrolment }\end{array}$ arm with HIV drug resistance

Staff perspectives regarding implementation During enrolment when point-of-care of point-of-care viral load testing in a routine testing is being implemented, and clinic after study conclusion

$\mathrm{ART}$, antiretroviral therapy.

ART drug level assays. These results would not be used to guide clinical management.

\section{Study outcomes}

We aim to broadly estimate the effect of point-of-care viral load testing using the primary outcome of viral suppression $<50$ copies/mL at 24 weeks after enrolment, measured on a laboratory reference assay (eg, Alinity $\mathrm{m} \mathrm{HIV-1)}$ in both arms. Feasibility outcomes include proportions of participants enrolled, followed up and who had same-day viral load testing in the point-of-care arm (table 2).

\section{Data collection and management}

The research team will capture study data using standardised electronic CRFs in REDCap, which is a password protected data management service stored on a secure server. ${ }^{18}$ All data entry will undergo three stages of quality control including preprogrammed data validity checks in electronic CRFs, immediate source document review 
and weekly quality reports generated using REDCap. To protect confidentiality, all study-specific documents, other than the signed consent, will refer to the participant using their study participant number rather than their name. All documents will be stored in a secure cabinet in the locked research office and will only be accessible by study staff and authorised personnel.

\section{Statistical methods}

At the conclusion of the study, we will assess the proportion of participants achieving study outcomes in each arm, with $95 \%$ CIs calculated using the Wilson method. We will conduct exploratory analyses using Fisher's exact test to compare the proportions achieving binary study outcome measures in each arm, using an intention to treat analysis. As this is a feasibility study, these exploratory analyses will likely not be powered to test the hypotheses that there is a difference between the two arms for most outcome measures.

\section{Primary outcome}

We will calculate the proportion of participants in each arm achieving the binary primary outcome of viral suppression $<50$ copies $/ \mathrm{mL}$ at 24 weeks (table 2). Participants who are lost to follow-up with no viral load result will be included in the analysis as not having achieved the primary outcome.

\section{Secondary outcomes}

We will calculate the proportion of potentially eligible patients with viraemia at the Prince Cyril Zulu clinic who are enrolled in the study (table 3). The denominator will be the number of patients with viraemia during the study enrolment period, which will be calculated by clinic staff using NHLS high viral load reports. These reports are routinely used for monitoring and evaluation of clinic performance and include the number of first high viral loads $\geq 1000$ copies/mL taken from the Prince Cyril Zulu Clinic each week. To assess study follow-up, we will calculate the proportion of participants who attend the study exit visit at 24 weeks after enrolment, overall and in each arm in case point-of-care testing influences retention. To assess implementation of point-of-care viral load testing, we will calculate the proportion of point-of-care viral load tests which are provided to participants on the same day, the proportion of viral loads $\geq 1000$ copies $/ \mathrm{mL}$ which resulted in same day enhanced adherence counselling, and the proportion of viral loads $\geq 1000$ copies $/ \mathrm{mL}$ resulting in appropriate same-day switch to second-line ART.

\section{Tertiary outcomes}

In each arm, we will assess the median number of days (and IQR) from enrolment to detection of viral failure, and from enrolment to appropriate switch to secondline ART (among participants with viral failure), and from enrolment to appropriate switching to dolutegravir (among participants on an NNRTI-based regimen). We will compare time to event outcomes using Cox proportional hazards. We will also calculate the proportion of participants with HIV drug resistance detected at 24 weeks after enrolment.

\section{Sample size considerations}

We conservatively estimate (based on our previous study) ${ }^{9}{ }^{10}$ that we could enrol approximately 100 participants in 6 months. If enrolment proceeds better than anticipated, and resources allow us to enrol more, this will provide more precise estimates of the study outcomes. If enrolment proceeds slower than expected, we will consider expanding to another clinic site to achieve a minimum of 80 participants. Therefore, while we anticipate 100 participants, the final number may be between approximately 80-180 participants, and will be determined by the time and resources available. We will use NHLS, Prince Cyril Zulu Clinic and study data to assess outcomes, with estimated precision, in table 3. Although not the primary aim of this feasibility study, assuming $50 \%$ of standard care participants achieve the main outcome of viral load suppression $<50$ copies/mL at 24 weeks, a sample size of 100 participants ( 50 per study arm) would provide $91 \%$ power to detect a $+30 \%$ difference in the point-of-care testing arm, using a two-sided alpha of 0.05 .

\section{Quality assurance procedures}

Study documentation will be subject to internal quality audits by the Centre for the AIDS Programme of Research in South Africa (CAPRISA) Quality Assurance team, in accordance with CAPRISA standard operating procedures.

\begin{tabular}{llll}
\hline Table 3 Study and process evaluation outcomes with precision estimates & & & \\
\hline Outcome & Estimated n/N, \% & 95\% Cl (Wilson) \\
\hline Percentage with viral load $<50$ copies/mL at 24 weeks in POC arm & $35 / 50,70.0$ & 56.2 to 80.9 & 36.6 to 63.4 \\
Percentage with viral load $<50$ copies/mL at 24 weeks in SOC arm & $25 / 50,50.0$ & 35.6 to 48.0 \\
Percentage of viraemic patients successfully enrolled in study & $100 / 240^{*}, 41.7$ & 82.6 to 94.5 \\
Percentage of those enrolled who are retained at 24 weeks & $90 / 100,90.0$ & 70.0 to 88.8 \\
Percentage in POC arm with same-day viral load testing & $40 / 50,79.5$ & \\
\hline
\end{tabular}

*Assuming 240 viraemic patients during enrolment period, many of whom will not be eligible due to being on second-line ART, having had previous high viral loads, already having received enhanced adherence counselling or pregnancy.

ART, antiretroviral therapy; POC, point-of-care; SOC, standard-of-care. 


\section{Qualitative substudy}

We will undertake a qualitative substudy within Point-Ofcare HIV viral load testing to Enhance Re-suppression (POwER) to assess what changes in clinic systems are required and what the views of staff are in implementing point-of-care viral load testing, its potential impact on adherence and adherence counselling and managing viraemia. We will conduct semistructured interviews and a focus group discussion with approximately 8-10 staff during, and then again after, implementation of point-ofcare viral load testing in the study. Staff will include counsellors, phlebotomists, nurses, pharmacy staff, laboratory staff, doctors and health service managers. Topic guides will be informed by normalisation process theory which aims to identify what is needed to 'normalise' use of a technology in a healthcare system. ${ }^{22}{ }^{23}$ Discussions will be transcribed and thematically analysed using NVIVO software (QSR International, Melbourne, Australia).

\section{Patient and public involvement}

Patients were not involved in the design of this study.

\section{Ethics and dissemination}

We will conduct the trial in accordance with the principles of the Declaration of Helsinki. The eThekwini Municipality Health Unit Research Committee, the KwaZulu-Natal Provincial Health Research Ethics Committee (KZ_202002_005), the University of KwaZulu-Natal Biomedical Research Ethics Committee (BREC/00000836/2019) and the University of Oxford Tropical Research Ethics Committee (OxTREC 66-19) have approved the study. We will present study results to front-line clinical workers and programme managers in eThekwini Municipality and KwaZulu-Natal Provincial Department of Health. We will also submit our findings to academic conferences and open-access, peer-reviewed journals.

\section{DISCUSSION}

Evidence-based interventions to improve the management of viraemia are needed to achieve global HIV treatment targets, prevent the development and spread of HIV drug resistance, and improve patient outcomes. In this paper, we outline a feasibility trial of point-of-care viral load testing among people with viraemia who are receiving first-line ART in South Africa. Results from this study will inform the design of future trials to assess the clinical effectiveness of point-of-care testing to manage viraemia.

\section{Problems with management of viraemia}

Several studies highlight gaps in the management of viraemia in LMICs, and the resulting negative impacts on treatment outcomes. First, addressing potential poor adherence, which may have caused viraemia, is challenging. A meta-analysis of interventions to improve adherence did not find any strategies that resulted in better viral suppression in LMICs, ${ }^{24}$ while a more recent cluster randomised trial of the South African National Adherence Guidelines found no difference in long-term viral resuppression among patients with viraemia. ${ }^{5}$ Second, follow-up viral load testing is often poorly performed. In the South African trial, less than $20 \%$ of patients had a repeat viral load within the recommended 3 months to confirm viral failure. ${ }^{5}$ Similar delays have been reported in a large analysis of South African laboratory data; among 260323 patients with viraemia, the median time to a repeat viral load was 6.2 months, (IQR 4.0-11.1) ${ }^{25}$ . Third, among people with confirmed viral failure, a systematic review, ${ }^{26}$ and more recent individual studies, ${ }^{4}{ }^{27-29}$ have found that only $30 \%-60 \%$ are switched to second-line ART. Even among patients who are switched, this can take a further 3-12 months, ${ }^{27-29}$ with delays of even a few months being associated with increased risk of opportunistic infections and mortality, particularly among people with low CD4 counts. ${ }^{1427-29}$ Taken together, these data highlight the need for interventions to reduce delays and improve the management of viraemia.

\section{Existing research regarding clinical effectiveness of point-of- care viral load testing}

We conducted the STREAM trial, which was the first randomised trial of point-of-care HIV viral load testing for monitoring ART. ${ }^{10}$ Overall, the STREAM intervention improved retention in care and viral suppression by $14.3 \%$ compared with the standardof-care laboratory viral load arm. However, STREAM was designed to enrol stable patients; less than $5 \%$ of participants had viraemia at enrolment, and only 16 participants developed viral failure (seven in the point-of-care arm, nine in the standard care arm). Time to detection of viral failure was faster in the intervention arm (55 days (IQR 55-57) vs 123 days (IQR 98-162)) but numbers with viraemia were too small to determine whether point-of-care testing was effective at improving switching to second line ART, or subsequent viral load outcomes. In STREAM, the research team provided point-of-care testing and clinical care, so the observed benefits may also not be applicable to routine public sector settings.

Several more trials of point-of-care viral load testing are in progress, but none focus on management of viraemia. Studies in Zimbabwe, ${ }^{30}{ }^{31}$ Kenya $^{32}$ and Haiti, ${ }^{33}$ are assessing point-of-care viral load for monitoring ART among pregnant women, children and/or adolescents. A study in Nigeria is enrolling adults at ART initiation and will report on the effect of pointof-care viral load testing after 12 months, ${ }^{34}$ while a trial in South Africa ${ }^{35}$ is enrolling adults on ART who are due for their annual viral load test. One trial in Uganda is assessing a viral load intervention package that includes 'near point-of-care testing' (at the clinic or a nearby clinic and therefore results may not be 
provided on the same day), and is enrolling 'high-risk groups' including patients with previous viraemia or no viral load in the past year. ${ }^{36}$

\section{Implementation of point-of-care viral load testing}

Point-of-care diagnostics have been used widely in healthcare systems in many LMICs. ${ }^{37}$ In HIV programmes, some tests such as rapid, lateral flow assays for diagnosis of HIV, have been evaluated, endorsed and incorporated into WHO guidelines and successfully adopted in many settings. ${ }^{38}$ However, other assays, such as more complex molecular PCR technologies for tuberculosis, have remained as laboratory tests despite being marketed as point-of-care assays. ${ }^{37}$ Given that the Xpert HIV-1 VL and m-PIMA assays use similar platforms and take over 1 hour for results to be available, they have also been criticised as not being implementable as true point-of-care tests. A large systematic review of barriers to point-of-care HIV diagnostic implementation in LMICs found that in 132 studies, integration of the point-of-care test into clinical work flows was the most commonly identified challenge to test utilisation. ${ }^{39}$ More recently, studies have reported implementation of qualitative point-of-care viral load assays for early infant diagnosis of HIV, ${ }^{40}$ and monitoring of ART in maternity wards in South Africa, ${ }^{41}$ and decentralised ART clinics in Malawi. ${ }^{42}$ While these studies have reported quantitative outcomes related to point-of-care viral load use, there is little qualitative work using theory-based approaches to evaluate what is required for successful implementation.

\section{Strengths and limitations}

A major strength of this study is the focus on patients with viraemia, who are a priority population at high risk of increased morbidity and mortality, onwards HIV transmission and development of HIV drug resistance. ${ }^{1}$ Interventions to improve viral suppression among this group have been prioritised by the WHO and align with several objectives in the Global Action Plan on HIV drug resistance. ${ }^{43}$ While our study may be underpowered to detect a significant effect of point-of-care viral load testing on viral resuppression, we will determine the feasibility of conducting larger studies to answer this question in this setting. Our use of normalisation process theory will allow a rigorous assessment of what is needed to 'normalise' point-ofcare testing in the South African healthcare system.

A limitation of this study is the focus on 'supply-side' implementation of point-of-care viral load testing. Receiving results on the same day could also increase patient understanding and motivate adherence, but evaluating this from the patient perspective is beyond the scope of the study. Furthermore, findings from this large urban, clinic may not be applicable to other settings, including smaller clinics in rural settings where advantages of point-of-care testing over laboratory-based testing may differ. Our future trial may be best designed as a cluster randomised trial, with individual clinics randomised to receive the point-of-care testing intervention. While POwER is an individually randomised study, estimates of the effect of point-of-care testing will remain valuable for design of potential cluster randomised or individually randomised future studies.

Coinciding with the rollout of dolutegravir is both a strength and a weakness of our study. We will enrol patients on either NNRTI- or dolutegravir-based ART, therefore, allowing estimates of the effect of point-ofcare testing in either group. For patients enrolled on NNRTIs, this also presents an opportunity to assess whether point-of-care viral load testing can assist the transition to dolutegravir-based regimens. For patients enrolled on dolutegravir, a potential limitation is that point-of-care viral load testing will not be used to expedite a switch to second line, although it may still improve enhanced adherence counselling and allow more efficient care that can help ensure retention and virological suppression. Lastly, we will be able to assess early viral suppression outcomes and the frequency of HIV drug resistance among people with viraemia on dolutegravir. This is a pressing research question for global policy makers, because it determines the need for HIV drug-resistance testing and second-line ART after introduction of a dolutegravir-based first-line in LMICs.

In summary, we aim to provide evidence as to whether a trial of point-of-care viral load testing to manage viraemia is feasible, and how testing may be implemented in South African clinics. This work will contribute to the development of interventions to improve the management of viraemia among people receiving ART, thereby improving health and leading to longer and better lives, as well as reducing the spread of HIV and preventing drug resistance.

\section{Author affiliations}

${ }^{1}$ Nuffield Department of Primary Care Health Sciences, University of Oxford, Oxford, UK

${ }^{2}$ Centre for the Aids Programme of Research in South Africa, University of KwaZuluNatal, Durban, South Africa

${ }^{3}$ eThekwini Municipality Health Unit, Durban, South Africa

${ }^{4}$ KwaZulu-Natal Research and Innovation Sequencing Platform (KRISP), University of KwaZulu-Natal, Durban, South Africa

${ }^{5}$ Department of Virology, University of KwaZulu-Natal, Durban, South Africa

${ }^{6}$ National Health Laboratory Service, Inkosi Albert Luthuli Central Hospital, Durban,

South Africa

${ }^{7} \mathrm{NIHR}$ Health Protection Research Unit in Healthcare Associated Infections and Antimicrobial Resistance, University of Oxford, Oxford, UK

${ }^{8}$ Department of Global Health, Schools of Medicine and Public Health, University of Washington, Seattle, Washington, USA

${ }^{9}$ Department of Medicine, School of Medicine, University of Washington, Seattle, Washington, USA

${ }^{10}$ Department of Epidemiology, School of Public Health, University of Washington, Seattle, Washington, USA

${ }^{11}$ Discipline of Public Health Medicine, School of Nursing and Public Health, University of KwaZulu-Natal, Durban, South Africa 
Twitter Richard Lessells @rjlessells and Christopher C Butler @ChrisColButler

Acknowledgements The authors would like to thank all participants in the study and acknowledge the work and support of staff at the Prince Cyril Zulu Clinic, eThekwini Municipality, CAPRISA and the National Health Laboratory Services at Addington and Inkosi Albert Luthuli Hospitals.

Contributors JD and NG conceived the study. YS, HN, RL, FS, EB, PM, NS, LL, ST-C, PKD, GH and CCB contributed to study design and implementation. ST-C contributed to design of the qualitative substudy. JD wrote the first draft of the manuscript. All authors critically reviewed and edited the manuscript and consented to final publication.

Funding This work is supported by grants from the Wellcome Trust PhD Programme for Primary Care Clinicians (216421/Z/19/Z), the University of Oxford's Research England QR Global Challenges Research Fund (0007365) and the Africa Oxford Initiative (AfiOx-119). The University of Oxford is the study sponsor.

Disclaimer The funders and sponsor have no role in study design, manuscript submission, or collection, management, analysis or interpretation of study data

Competing interests Cepheid and Abbott have provided point-of-care viral load assays at no cost for use at the study site.

Patient consent for publication Not required.

Provenance and peer review Not commissioned; externally peer reviewed.

Supplemental material This content has been supplied by the author(s). It has not been vetted by BMJ Publishing Group Limited (BMJ) and may not have been peer-reviewed. Any opinions or recommendations discussed are solely those of the author(s) and are not endorsed by BMJ. BMJ disclaims all liability and responsibility arising from any reliance placed on the content. Where the content includes any translated material, BMJ does not warrant the accuracy and reliability of the translations (including but not limited to local regulations, clinical guidelines, terminology, drug names and drug dosages), and is not responsible for any error and/or omissions arising from translation and adaptation or otherwise.

Open access This is an open access article distributed in accordance with the Creative Commons Attribution 4.0 Unported (CC BY 4.0) license, which permits others to copy, redistribute, remix, transform and build upon this work for any purpose, provided the original work is properly cited, a link to the licence is given, and indication of whether changes were made. See: https://creativecommons.org/ licenses/by/4.0/.

\section{ORCID iDs}

Jienchi Dorward http://orcid.org/0000-0001-6072-1430

Sarah Tonkin-Crine http://orcid.org/0000-0003-4470-1151

Gail Hayward http://orcid.org/0000-0003-0852-627X

Christopher C Butler http://orcid.org/0000-0002-0102-3453

Nigel Garrett http://orcid.org/0000-0002-4530-234X

\section{REFERENCES}

1 Murphy RA, Court R, Maartens G, et al. Second-Line antiretroviral therapy in sub-Saharan Africa: it is time to mind the gaps. AIDS Res Hum Retroviruses 2017;33:1181-4.

2 Labhardt ND, Ringera I, Lejone TI, et al. When patients fail UNAIDS' last 90 - the "failure cascade" beyond 90-90-90 in rural Lesotho, Southern Africa: a prospective cohort study. J Int AIDS Soc 2017;20:21803-10.

3 Etoori D, Ciglenecki I, Ndlangamandla M, et al. Successes and challenges in optimizing the viral load cascade to improve antiretroviral therapy adherence and rationalize second-line switches in Swaziland. J Int AIDS Soc 2018;21:e25194-e94.

4 Hermans LE, Carmona S, Nijhuis M, et al. Virological suppression and clinical management in response to viremia in South African HIV treatment program: a multicenter cohort study. PLoS Med 2020;17:e1003037.

5 Fox MP, Pascoe SJS, Huber AN, et al. Effectiveness of interventions for unstable patients on antiretroviral therapy in South Africa: results of a cluster-randomised evaluation. Trop Med Int Health 2018;23:1314-25.

6 Shubber Z, Mills EJ, Nachega JB, et al. Patient-Reported barriers to adherence to antiretroviral therapy: a systematic review and metaanalysis. PLoS Med 2016;13:e1002183-e83.

7 World Health Organization. WHO prequalification of in vitro diagnostics. Product : Xpert@ HIV-1 Viral Load with GeneXpert $®$ Dx,
GeneXpert $\circledast$ Infinity- 48, GeneXpert $\circledast$ Infinity-48s and GeneXpert $\circledast$ Infinity-80; 2017.

8 World Health Organization. WHO prequalification of in vitro diagnostics public report. Product: m-PIMA HIV-1/2 VL; 2019

9 Dorward J, Garrett N, Quame-Amaglo J, et al. Protocol for a randomised controlled implementation trial of point-of-care viral load testing and task shifting: the Simplifying HIV TREAtment and Monitoring (STREAM) study. BMJ Open 2017;7:e017507-e07.

10 Drain PK, Dorward J, Violette LR, et al. Point-Of-Care HIV viral load testing combined with task shifting to improve treatment outcomes (STREAM): findings from an open-label, non-inferiority, randomised controlled trial. Lancet HIV 2020;7:e229-37.

11 Mody A, Geng EH. Conceptualising implementation strategies in HIV research. Lancet HIV 2020;7:e382.

12 Eldridge SM, Lancaster GA, Campbell MJ, et al. Defining feasibility and pilot studies in preparation for randomised controlled trials: development of a conceptual framework. PLoS One 2016;11:e015 0205-e05.

13 South Africa National Department of Health. National consolidated guidelines for the management of HIV in adults, adolescents, children and infants and prevention of mother-to-child transmission. Pretoria, South Africa: South African National Department of Health, 2020.

14 Dorward J, Lessells R, Drain PK, et al. Dolutegravir for first-line antiretroviral therapy in low-income and middle-income countries: uncertainties and opportunities for implementation and research. Lancet HIV 2018;5:e400-4.

15 Vitoria M, Hill A, Ford N, et al. The transition to dolutegravir and other new antiretrovirals in low-income and middle-income countries: what are the issues? AIDS 2018;32:1551-61.

16 Hamers RL, Rinke de Wit TF, Holmes CB. HIV drug resistance in lowincome and middle-income countries. Lancet HIV 2018;5:e588-96.

17 World Health Organization. Consolidated guidelines on the use of antiretroviral drugs for treating and preventing HIV infection: recommendations for a public health approach. 2nd edn. Geneva, Switzerland, 2016: 427-27.

18 Harris PA, Taylor R, Thielke R, et al. Research electronic data capture (REDCap)--a metadata-driven methodology and workflow process for providing translational research informatics support. J Biomed Inform 2009;42:377-81.

19 South Africa National Department of Health. Minimum package of interventions to support linkage to care, adherence and retention in care: South African National Department of Health; 2020.

20 The South African National Department of Health. 2019 ART clinical guidelines for the management of HIV in adults, pregnancy, adolescents, children, infants and neonates. Pretoria, South Africa, 2019.

21 National Health Research Ethics Council. Payment of trial participants in South Africa: ethical considerations for research ethics committees: South African Department of Health; 2012.

22 May C, Finch T, Implementing FT. Implementing, embedding, and integrating practices: an outline of normalization process theory. Sociology 2009;43:535-54.

23 May CR, Mair F, Finch T, et al. Development of a theory of implementation and integration: normalization process theory. Implement Sci 2009;4:29.

24 Kanters S, Park JJH, Chan K, et al. Interventions to improve adherence to antiretroviral therapy: a systematic review and network meta-analysis. Lancet HIV 2017;4:e31-40.

25 Fox MP, Brennan AT, Nattey C, et al. Delays in repeat HIV viral load testing for those with elevated viral loads: a national perspective from South Africa. J Int AIDS Soc 2020;23:e25542.

26 Ford N, Orrell C, Shubber Z, et al. HIV viral resuppression following an elevated viral load: a systematic review and meta-analysis. J Int AIDS Soc 2019;22:1-6.

27 Rohr JK, Ive P, Horsburgh CR, et al. Marginal structural models to assess delays in second-line HIV treatment initiation in South Africa. PLoS One 2016;11:e0161469-11.

28 Bell-Gorrod H, Fox MP, Boulle A, et al. The impact of delayed switch to second-line antiretroviral therapy on mortality, depending on definition of failure time and CD4 count at failure. Am J Epidemiol 2020;189:811-9.

29 Bell Gorrod H, Court R, Schomaker M, et al. Increased mortality with delayed and missed switch to second-line antiretroviral therapy in South Africa. J Acquir Immune Defic Syndr 2020;84:107-13.

30 Munyati S. NCT03986099 Community based antiretroviral therapy (CBART) among children on chronic ART, 2019. Clinicaltrials. Gov. Available: https://clinicaltrials.gov/ct2/show/NCT03986099 [Accessed 28 Aug 2020].

31 Clinton Health Access Initiative. NCT04048629 impact of point-ofcare (POC) viral load (VL) testing during pregnancy in Zimbabwe, 
2019. Clinicaltrials.Gov. Available: https://clinicaltrials.gov/ct2/show/ NCT04048629 [Accessed 28 Aug 2020].

32 Patel R. NCT03820323 Optimizing viral load suppression in Kenyan children on antiretroviral therapy (Opt4Kids), 2019. Clinicaltrials. Gov. Available: https://clinicaltrials.gov/ct2/show/NCT03820323 [Accessed 28 Aug 2020].

33 Reif LK, Belizaire ME, Seo G, et al. Point-Of-Care viral load testing among adolescents and youth living with HIV in Haiti: a protocol for a randomised trial to evaluate implementation and effect. BMJ Open 2020;10:e036147.

34 Meloni ST, Agbaji O, Chang CA, et al. The role of point-of-care viral load monitoring in achieving the target of $90 \%$ suppression in HIV-infected patients in Nigeria: study protocol for a randomized controlled trial. BMC Infect Dis 2019;19:1-11.

35 Theron G. NCT03187964: Xpert ultra and Xpert HIV-VL in people living with HIV (UltraHIV), 2017. Clinicaltrials.Gov. Available: https://clinicaltrials.gov/ct2/show/NCT03187964[Accessed 10 Feb 2021].

36 Jain V. NCT03553693: rapid HIV viral load monitoring in high risk patients in Uganda (RAPID-VL), 2018. Clinicaltrials.Gov. Available: https://clinicaltrials.gov/ct2/show/NCT03553693 [Accessed 10 Feb 2021].
37 Jani IV, Peter TF. How point-of-care testing could drive innovation in global health. N Engl J Med 2013;368:2319-24.

38 Drain PK, Rousseau C. Point-Of-Care diagnostics: extending the laboratory network to reach the last mile. Curr Opin HIV AIDS 2017;12:175-81.

39 Pai NP, Wilkinson S, Deli-Houssein R, et al. Barriers to implementation of rapid and point-of-care tests for human immunodeficiency virus infection: findings from a systematic review (1996-2014). Point Care 2015;14:81-7.

40 Bianchi F, Cohn J, Sacks E, et al. Evaluation of a routine point-ofcare intervention for early infant diagnosis of HIV: an observational study in eight African countries. Lancet HIV 2019;6:e373-81.

41 Kufa T, Mazanderani AH, Sherman GG, et al. Point-Of-Care HIV maternal viral load and early infant diagnosis testing around time of delivery at tertiary obstetric units in South Africa: a prospective study of coverage, results return and turn-around times. $J$ Int AIDS Soc 2020;23:e25487.

42 Nicholas S, Poulet E, Wolters L, et al. Point-of-care viral load monitoring: outcomes from a decentralized HIV programme in Malawi. J Int AIDS Soc 2019;22:1-9.

43 World Health Organization. Global action plan for HIV drug resistance 2016-2021. Geneva, Switzerland, 2017. 